MATEC Web of Conferences 40, 08003 (2016)

DOI: $10.1051 /$ matecconf/20164008003

(C) Owned by the authors, published by EDP Sciences, 2016

\title{
Permanent magnet brushless motor control based on ADRC
}

\author{
Xiaokun $\mathrm{Li}^{1}$, Song Wang ${ }^{1}$, XiaoFan Wang ${ }^{1}$, Tingting Shi ${ }^{1}$ \\ ${ }^{1}$ School of Mechanical, Electrical \& Information Engineering, Shandong University (Weihai), Weihai, China
}

\begin{abstract}
Permanent magnet brushless motor is a nonlinear system with multiple variables, the mathematical model of Permanent magnet brushless motor is difficult to establish, and since that the classic PID control is hard to precisely control the motor. Active disturbance rejection control (ADRC) technique is a new nonlinear controller which does not depend on the system model. It is starting from the classic PID control, and establishing the loop control system by error negative feedback, the ESO(extended state observer) observing system which comes from the observer theory of modern control theory to observe internal and external perturbations. ADRC inherits the advantages of PID with little overshoot, high convergence speed, high accuracy, strong anti-interference ability and other characteristics, and it has a strong disturbance adaptability and robustness as for the uncertainty perturbation and their internal disturbance of control objects. Therefore, This paper attempts to use Active disturbance rejection control(ADRC), in order to improve the control of permanent magnet brushless motor. In this design of control system, the simulation of the system is realized based on MATLAB, and then the discrete control algorithm is transplanted to the embedded system to control the permanent magnet brushless DC motor (PMBLDCM). The control system is implemented on the DSP-F28335 digital signal processor, and the DSP also provides the functions like voltage and current AD sampling, PWM driver generation, speed and rotor position calculation, etc. The simulation and experiment results indicate that, the system has good dynamic performance and anti-disturbance performance. Key words: Permanent magnet brushless motor; Active disturbance rejection control; MATLAB; DSP-F28335
\end{abstract}

\section{Introduction}

Permanent magnet brushless DC motor (PMBLDCM) has the advantage of $\mathrm{AC}$ motor, such as the simple structure, reliable operation, easy maintenance, etc. Furthermore, it is also with high efficiency, speed performance and other characteristics of DC motor. With the rapid development of modern power electronics and computer technology, it has a wider application in the aviation, aerospace, defense, and all areas of daily life [1].

PMBLDCM uses power electronic devices consisting of an electronic commutator instead of mechanical commutation of DC brush motor. Permanent magnet brushless motor has excellent speed performance like DC motor since its stator field and rotor are decoupled [2]. Moreover, the rotor has the advantage of AC induction motor like simple rotor structure, reliable operation, convenient maintenance, etc. Since permanent magnet rotor of permanent magnet brushless motor can not be adjusted, the output torque and speed can only be adjusted by reducing the stator voltage. Speed is mainly controlled by regulating the stator voltage through the way of PWM [3]. The control of closed-loop speed is still using the classic method of PID, PID controller has simple structure and is easy to adjust the parameters. In most cases, PID control has been able to meet the control needs, but for the permanent magnet brushless motor, because of its multi-variable, strong nonlinear and poor controlling effect, the PID control technology is only applicable to low accuracy needs.

At present, many new control algorithms have been proposed, such as neural network control, intelligent optimization algorithm[4, 5], fuzzy logic control and other control methods in domestic and abroad. They have made some progress but do not have further applications since the complexity design. Such as model reference adaptive control is difficult to adapt to dramatic changes in load; the control effect state of fuzzy control is not ideal due to the complex interactions of fuzzy rules; while the neural network controller requires relatively large resources, the hardware implementation is arduous [6]. ADRC is a state observer combination product of classic error PID feedback control and modern control theory, estimate system disturbances and to revise the error by system control output and the output feedback from the control object. This paper attempts to use active disturbance rejection control (ADRC) to research PMBLDCM control system.

\section{Active disturbance rejection control}

The ADRC technology is derived from the PID and modern control theory. The ADRC has higher stability [7-9] and robustness than the PID controller and thus has 
been used to address the inherent defects of classical PID [10].

\subsection{ADRC controller}

The ADRC consists of two parts. The first part is the tracking differentiator (TD), which tracks the system reference input and reasonably arranges the transient process. The second part is the extended state observer (ESO), which controls the system, observes the "integrated disturbance" of systems through the nonlinear-state error feedback (NLSEF) generalized error, and provides the corresponding feedforward compensation of the disturbance [11].

The ADRC can be widely used for the unknown disturbances of nonlinear uncertain systems[12], as follows:

$$
\left\{\begin{array}{l}
x^{(n)}=f\left(x, \dot{x}, \ldots, x^{(n-1)}, t\right)+\omega(t)+b u \\
y=x(t)
\end{array}\right.
$$

Where $f\left(x, \dot{x}, \ldots, x^{(n-1)}, t\right)$ is the unknown function, $\omega(t)$ is the unknown disturbance, $x(t)$ is the system output (which can be measured), $u$ is the control action of the system, and $b$ is the gain.

\subsection{The mathematical model of $\mathrm{n}$ order ADRC} (1)

The composition of ADRC can be shown as equation

(a) N-order tracking differentiator (TD)

The n-order tracking differentiator of nonlinear system is usually in the form of equation (2):

$$
\left\{\begin{array}{l}
\dot{z}_{1}=z_{2} \\
\dot{z}_{2}=z_{3} \\
\cdots \cdots \\
\dot{z}_{n}=R^{n} f\left(z_{1}-v(t), \frac{z_{2}}{R}, \cdots \frac{z_{n}}{R^{n-1}}\right)
\end{array}\right.
$$

Where $z_{1}$ is the tracking signal of given signal $v(t)$, $\dot{z}_{2} \cdots \cdots \dot{z}_{n}$ is the derivative of different order of $v(t)$, and R is the boundary of the TD input signal.

(b) Extended state observer (ESO)

As for the nonlinear system as equation(2) shown, the general form of $n+1$ order ESO can be shown as equation (3) :

$$
\left\{\begin{array}{l}
\dot{z}_{1}=z_{2}-g_{1}\left[z_{1}-x(t)\right] \\
\cdots \cdots \\
\dot{z}_{n}=z_{n+1}-g_{n}\left[z_{1}-x(t)\right]+b u \\
\dot{z}_{n+1}=-g_{n+1}\left[z_{1}-x(t)\right]
\end{array}\right.
$$

Where $z_{1}$ is the tracking signal of system output, $\dot{z}_{1}, \dot{z}_{2} \cdots \cdots \dot{z}_{n}$ is the derivative of different order of output, $\dot{z}_{n+1}$ is the real-time estimation of the total disturbance- $a(t)$, and $a(t)$ can be shown as follows:

$$
a(t)=f\left(x, \dot{x}, x^{(n-1)}, t\right)+w(t)
$$

\section{(c) Nonlinear State Error Feedback (NLSEF)}

Unlike the weighted way in PID control, nonlinear combination of errors is always used in ADRC control. The weight is revised nonlinearly according to different errors ranges, to achieve better results than PID control. The commonly used non-linear combination functions can be seen as follows:

$$
\begin{gathered}
u=\beta_{0} \mathrm{fal}\left(e_{0}, a, \delta\right)+\beta_{1} \mathrm{fal}\left(e_{1}, a, \delta\right)+\beta_{2} \mathrm{fal}\left(e_{2}, a, \delta\right) \\
u=\beta_{0} e_{0}+\operatorname{fhan}\left(e_{1}, c^{*} e_{2}, r, h_{0}\right)
\end{gathered}
$$

where

$$
\begin{aligned}
& \text { fhan }=-r[a / d-\operatorname{sign}(a)] s_{a}-r \operatorname{sign}(a) ; \\
& d=r h^{2} ; a_{0}=h x_{2} ; y=x_{1}+a_{0} ; \\
& a_{1}=\sqrt{d(d+8|y|)} ; a_{2}=a_{0}+\operatorname{sign}(y)\left(a_{1}-d\right) / 2 ; \\
& s_{y}=[\operatorname{sign}(y+d)-\operatorname{sign}(y-d)] / 2 ; \\
& a=\left(a_{0}+y-a_{2}\right) s_{y}+a_{2} \\
& s_{a}=[\operatorname{sign}(a+d)-\operatorname{sign}(a-d)] / 2 .
\end{aligned}
$$

When applying nonlinear combination, the parameters $a_{i}$ are generally following the range of : $a_{0}<0<a_{1}<1<a_{2} \beta_{1}$ and $\beta_{2}$ are used to adjust the proportional and differential effects on output. Usually $\beta_{1}$ is larger than $\beta_{2}$, and $\beta_{2}$ is used to suppress the shock when $\beta_{1}$ is excessive, whereas too large $\beta_{2}$ can suppress the output response. The coefficients of nonlinear combination can affect the output of the system, the amount of first-order differential is required to less than the disturbance component and the disturbance component is less than the tracking of input usually.

The main role of $\beta_{3}$ is to pass the interfere to ESO and then compute the amount of disturbance effects in real time, and then the feedback is passed to the output of control system by $z_{3}$-the output of extended state observer (ESO). Although $\beta_{3}$ only regulating $z_{2}$ apparently, $\beta_{3}$ can change the three outputs since $z_{1}, z_{2}, z_{3}$ have the differential relation, which are the most difficult to adjust in ADRC. 


\subsection{The discrete of ADRC}

The discrete equations of 2-order ADRC algorithmtracking differentiator (TD), extended state observer (ESO) and nonlinear-state error feedback (NLSEF) are shown as follows:

(a) 2-order TD discrete algorithm:

$$
\left\{\begin{array}{l}
v_{1}(k+1)=v_{1}(k)+h v_{2}(k) \\
v_{2}(k+1)=v_{2}(k)+h \operatorname{hhan}\left(v_{1}-v_{0}, v_{2}, r, h\right)
\end{array}\right.
$$

(b) 3-order ESO discrete algorithm:

$$
\left\{\begin{array}{l}
e=z_{1}(k)-y(k) \\
z_{1}(k+1)=z_{1}(k)+h\left(z_{2}(k)-\beta_{1} e\right) \\
z_{2}(k+1)=z_{2}(k)+h\left[z_{3}(k)-\beta_{2} f a l\left(e, a_{1}, \delta\right)+b u(k)\right] \\
\left.z_{3}(k+1)=z_{3}(k)-h \beta_{3} f a l\left(e_{1}, a_{2}, \delta\right)\right]
\end{array}\right.
$$

(c) NLSEF discrete algorithm

$$
\left\{\begin{array}{l}
e_{1}=v_{1}(k)-z_{1}(k) \\
e_{2}=v_{2}(k)-z_{2}(k) \\
u_{0}=\beta_{1} f a l\left(e_{1}, a_{1}, \delta\right)+\beta_{2} f a l\left(e_{2}, a_{2}, \delta\right) \\
u(k)=u_{0}-\frac{z_{3}(k)}{b}
\end{array}\right.
$$

Where $h$ is the sampling period. This will be used for experiments.

\section{The simulation of motor controlled system based on ADRC}

The simulation model of PMBLDCM servo control system based on ADRC is established using the SimPower System Toolbox in Matlab-Simulink environment. 2-order ADRC control model includes tracking differentiator (TD), extended state observer (ESO) and nonlinear-state error feedback (NLSEF). Then ADRC control algorithm combined with BLDC motor and inverter are realized in Simulink to build a completely motor control system [13]. The control system diagram of BLDCM is shown in figure 1, and Simulink model of BLDCM is shown in figure 2.

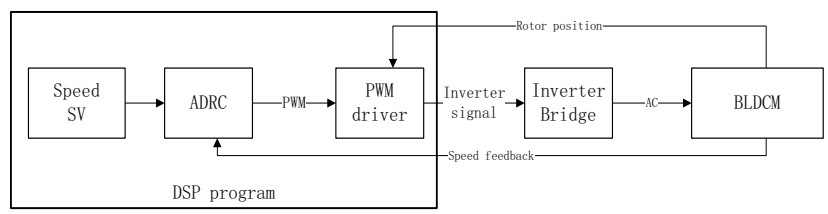

Fig.1 The control system diagram of BLDCM

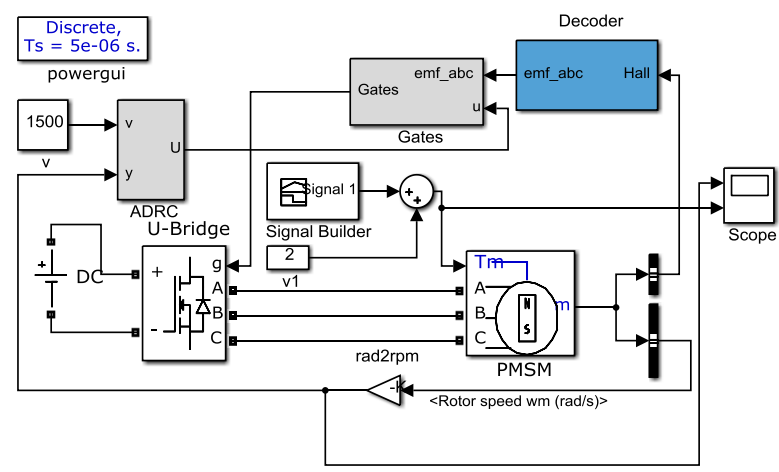

Fig2. The simulation of BLDCM in Simulink

ADRC is the controller in this model, its inputs, refers to the system set speed $\mathrm{v}=1500$ and the $\mathrm{y}$ is the speed output of PMBLDCM; $u$ is the output regulating by PWM duty cycle. The encoder is responsible for detecting the rotor position. The rotation angle of motor rotor can be judged through the signal changes of Hall sensor when rotor rotates.

\subsection{Tracking differentiator (TD)}

Tracking differentiator is mainly to soften the control signal. The parameter $r$ can be determined, based on the requirements of the actual application transition process. If $v_{1}$, the output signal of TD, can accurately track the input signal $v$, then the TD parameter is appropriate. The larger $r$ is, the shorter the process that $v_{1}$ reaches the input signal $v$, but the controlled object may not be able to achieve the default value in a short time. As for PWM speed-controlling motor, the tracking differentiator should be obtained the maximum rising speed corresponding to the maximum value of $v_{2}$ when the PWM duty cycle of motor is $80 \%$.

In order to achieve "Fast and optimal control", the second-order equation of ADRC control algorithm can be shown as:

$$
\left\{\begin{array}{l}
\dot{v}_{1}=v_{2} \\
\dot{v}_{2}=-r \operatorname{sgn}\left(v_{1}-v(t)+v_{2}\left|v_{2}\right| /(2 r)\right)
\end{array}\right.
$$

Where $r$ determines the tracking speed, it can be verified according to the model building in Simulink. The system input is $\mathrm{r}$, setting speed is $\mathrm{v}$ and outputs are $\mathrm{yl}$ and y2. Refers to first order and second-order of the trace output respectively, the mathematical model is shown as figure 3 :



Fig.3 The mathematical model of TD 
Tracking differential effect can be different with different $r$ as figure 4, with consistent the scope parameter, the figure 4 (a) $\mathrm{r}=2000$, the input $\mathrm{v}=1500$ and figure 4(b) $\mathrm{r}=5000$, the input $\mathrm{v}=1500$. The results indicate: parameter $r$ impacts the tracking speed and maximum second-order derivative, the greater $r$ is, the faster tracking speed is, and draw a large differential value, the smaller $r$ is, the slower tracking speed and the smaller differential value is.

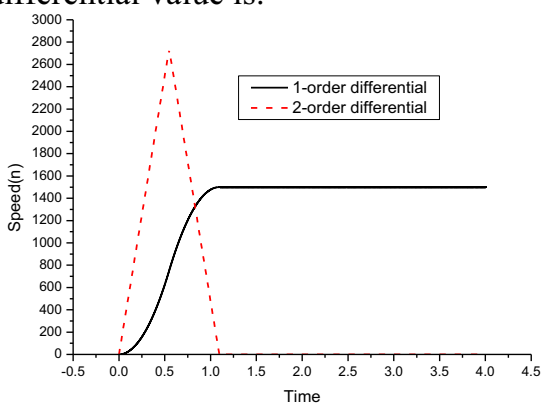

(a) $r=2000$

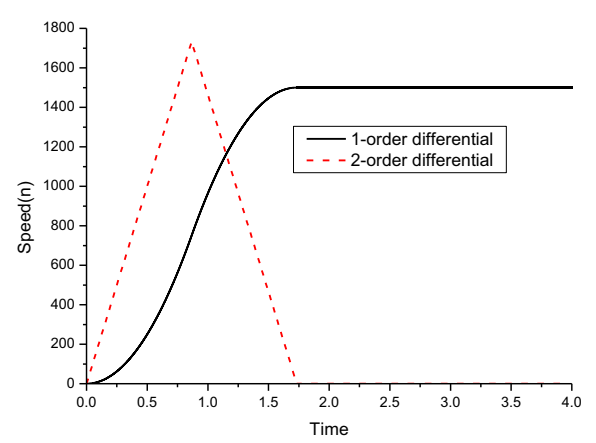

(b) $r=5000$

Fig.4 Tracking differential effect with different $r$

It can also be seen that tracking differential module only affects the first order and second-order error of the transition process, and it will no longer work after the transition process.

\subsection{Extended state observer (ESO)}

The tuning of ESO is firstly used to ensure that $\mathrm{zl}$ can trace output $y$ precisely, then adjust the parameter of disturbance estimation. Disturbance estimated gain $b$ is passed to the differential of output feedback; accordingly the tracking of the output feedback will be affected, so that repeatedly adjusting is needed to achieve parameters co-ordination.

Disturbance estimation mainly depends on $\beta_{3}$, the greater $\beta_{3}$ is, the violent reaction of the disturbance will cause rapid oscillation as to system output, thus the load characteristics is considered when choosing $\beta_{3}$. Parameter $b$ reflects the speed of system eliminating static error, and the smaller $b$ is the faster elimination of static error. However, it can also cause the system oscillation, and the appropriate parameters need repeatedly adjust.
The equation of second-order ESO can be shown below:

$$
\left\{\begin{array}{l}
e=z_{1}-y \\
\dot{z}_{1}=z_{2}-\beta_{01} e \\
\dot{z}_{2}=z_{3}-\beta_{02} e+b u \\
\dot{z}_{3}=-\beta_{03} e
\end{array}\right.
$$

The simulink model is established in accordance with the equation and shown in Figure 5 :

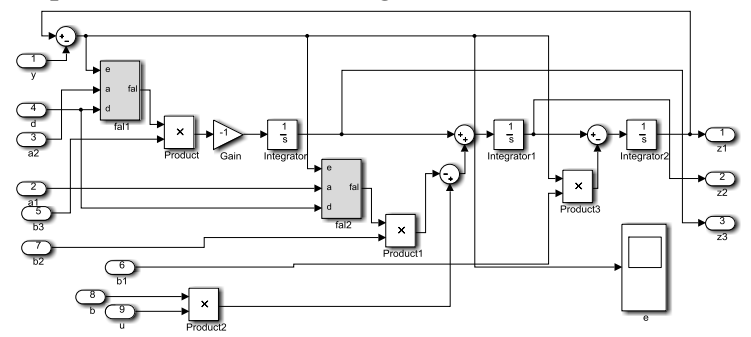

Fig.5 Simulink model of ESO

$\mathrm{z} 1$ is mainly debugged to trace the changes of output $\mathrm{y}$ in ESO, the second-order differential of output feedback $\mathrm{z} 2$ and the internal and the external disturbance of system $\mathrm{z} 3$ are calculated by $\mathrm{u}$ and $\mathrm{y} . \mathrm{z} 3$ can good estimate "the action in real time" of system disturbances.

\subsection{Anti-interference simulation of ADRC}

ADRC of speed loop is firstly used to track the differential input settings, getting default the speed tracking value v1 and the second-order derivative value v2, then gives the observer of controller status feedback of the motor speed and controlling output, and then the current output tracking $\mathrm{z} 1, \mathrm{z} 2$ and differential perturbation component $\mathrm{z} 3$ are calculated. In the NLSEF, $\mathrm{z} 1$ and v1are compared, and the differential z2 and the differential input $\mathrm{z} 2$ are compared accordingly to the error e 1 and $\mathrm{e} 2$, and the output of controller is calculated. Through the nonlinear combination e1, e2 and $\mathrm{z} 3$, a closed-loop control system is realized [14]. The algorithm diagram is shown in figure 6 :

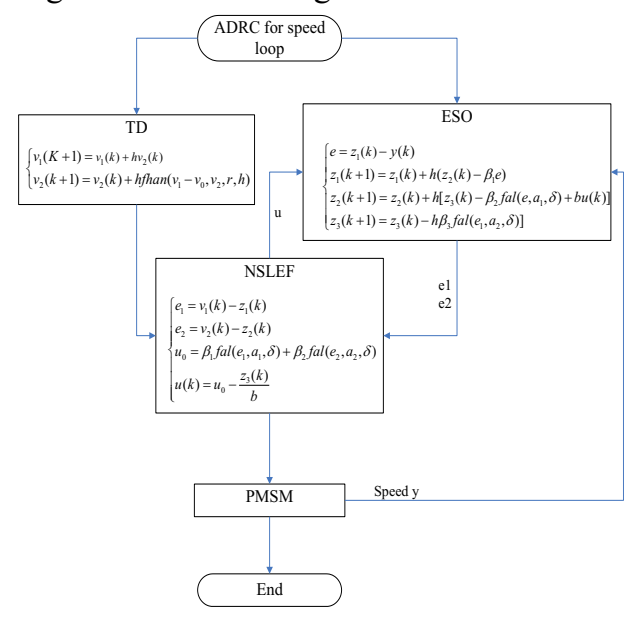

Fig.6 Algorithm block diagram of ADRC 
The ADRC diagram in Simulink is shown in Figure7. The system parameters are as follows:

The step of system: $h=5 e^{-6}$

Tracking differentiator (TD): $r=5000$

Extended state observer (ESO):

esoa $1=0.5 ;$ esoa $2=0.25: d=0.01$;

$e s o b 1=2000 ;$ esob $2=1000 ;$ esob3 $=200 ; b=10$

Nonlinear-state error feedback (NLSEF):

ctral $=0.6 ; c t r a 2=0.4 ; c t r b 1=50 ; c t r b 2=2 ; d=0.01$

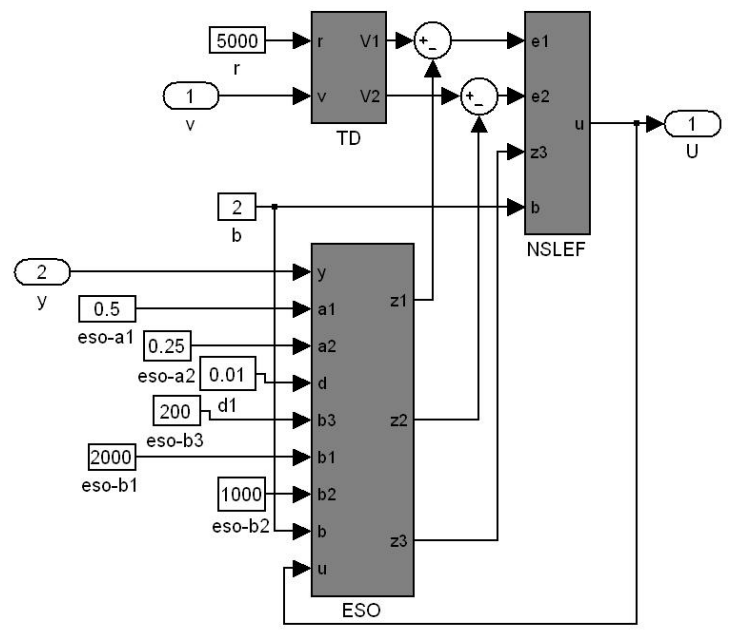

Fig.7 The simulation diagram of ADRC in Simulink

The starting torque of motor is $2 \mathrm{NM}$ and then adding interference torque $2 \mathrm{NB}$ at $2 \mathrm{~s}$, the motor speed response is shown in figure 8:

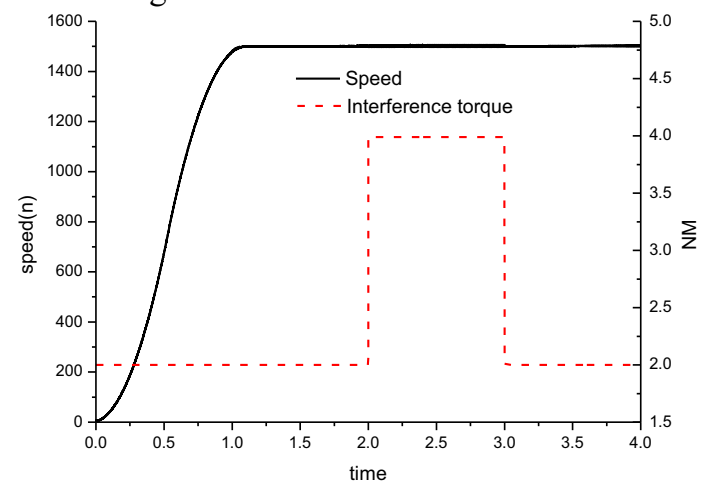

Fig.8 Speed and torque graph of PMBLDCM

In order to testify the anti-disturbance capability of the system, a square wave (torque $=4 \mathrm{NM}$, width $=1$ ) interference is added at $\mathrm{t}=2 \mathrm{~s}$, the reaction of system is shown in figure 9. As it can be seen in the figure above, the response of control system is very fast. In the case that the motor speed is in the disturbance, load torque does not vibrate a lot when increasing from $2 \mathrm{NM}$ to $4 \mathrm{NM}$. Observing perturbations details after enlarging can be seen in Figure 9: the disturbance is eliminated when $\mathrm{t}=$ $3 \mathrm{~s}$, it is clear that the motor speed quickly becomes stabilized, from when the load torque is $4 \mathrm{NM}$ and speed error is about $10 \mathrm{rpm}$ to when the load is $2 \mathrm{NM}$ and speed error is about 2rpm (1501-1502). The output of motor speed fluctuates a lot when the torque is large, but it can remain stable, the speed fluctuation will quickly become smaller after the torque becomes smaller.



Fig.9 Disturbance on the system speed graph

Through the analysis of the model we can know, TD and ESO can ensure the ability of tracking the input $\mathrm{v}$ and output y respectively, and by adjusting the nonlinear-state error feedback (NLSEF), the output will match the controlled object, and finally further to optimize each parameters of ESO to get a better control effect. Nonlinear-state error feedback (NLSEF) is directly related to the amount of output control, it has a great impact on the output waveform, such as the PWM control, inappropriate nonlinear-state error feedback (NLSEF) parameter settings can easily result in the controlling value of output being not less than 0 or greater than the amount 100 , losing the ability to control speed, while controlling amount will repeatedly vibrate between 0 and 100. The tuning process of Active disturbance rejection control (ADRC) control is complicated than PID, but in the case of interference, the control effects is much better than PID.

\section{Experiment}

TI TMS320F28335 DSP is adopted as the hardware platform for signal processing and control. The inverter adopts the type of driver chip and MOSFET, and the motor is powered by $24 \mathrm{~V}$. The main circuit topology of motor control is shown in figure 10 .

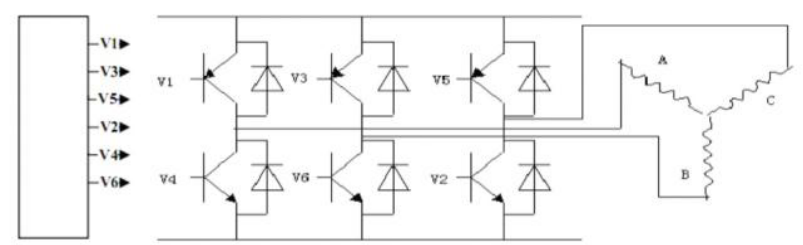

Fig. 10. The main circuit topology of motor control

The control signal output of inverter bridge, which originated from the ePWM interface of TMS320F28335 DSP to the driver chip, generates gate turn-on signals to drive the MOSFET inverter bridge. The driver chips are integrated isolation IR2136. The experiment system is shown in figure 11. 


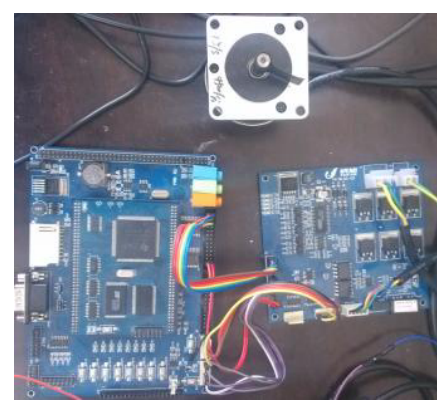

Fig. 11. Experiment system

In order to compare the PID with ADRC, different PID parameters are adopted to control the motor. When $\mathrm{Kp}=0.1$ and $\mathrm{Ki}=0.001$, the overshoot and shocks is big. The motor speed response changes when $\mathrm{Kp}=0.1$ and $\mathrm{Ki}=0.01$. Although the performance of PID is better by regulating the parameters, it is worse than the ADRC performance. There is no overshoot when the parameters of the ADRC are carefully adjusted. The motor speed fluctuates between 600-601 rpm. The performance contrast of PID and ADRC is shown in Figure 12.



Fig. 12 System response by PID and ADRC control

\section{Conclusion}

The 2-order simulink model of the anti-rejection controller is established by MATLAB $\backslash$ simulink and a permanent magnet brushless motor servo control system is realized to simulate and prove the effects of ADRC. Based on DSP28335 development board, the permanent magnet brushless motor control system has been designed, including basic circuit and permanent magnet brushless motor rotor positioning, speed detection, voltage/current sensing and transplantion of ADRC control algorithms. The permanent magnet brushless DC motor realized smooth start and governor by ADRC.

\section{Conflict of Interests}

The authors declare that there is no conflict of interests regarding the publication of this article.

\section{References}

1. B. Hao, J. Cui and X. Xu, "Research and Prospect of Permanent Magnet Brushless DC (PMBLDC) Motor Controller", MECHANICAL \& ELECTRICAL ENGINEERING MAGAZINE, vol. 21, No. 4, pp. 59-61, 2004.
2. W. Hu, "Developments and Prospects of Permanent Magnetic Brushless DC Motor", MICROMOTORS SERVO TECHNIQUE, vol. 35, No. 4, pp. 37-38, 2002.

3. P. TANG, Z. LIAO and T. LI, "Double-frequency PWM control scheme for BLDCM", ELECTRIC MACHINES AND CONTROL, vol. 13, No. 3, pp. 389-392,397, 2009.

4. Q. Xu, C. Zhang and L. Zhang, "A Fast Elitism Gaussian Estimation of Distribution Algorithm and Application for PID Optimization", The Scientific World Journal, vol. 2014, No. pp. 14, 2014.

5. Q. Xu, C. Zhang, L. Zhang and C. Wang, "Multiobjective Optimization of PID Controller of PMSM", Journal of Control Science and Engineering, vol. 2014, No. pp. 9, 2014.

6. Q. Yang, X. Hao and H. Zhang, "A New Controller of Brushless DC Motors", PROCESS AUTOMATION INSTRUMENTATION, vol. 29, No. 9, pp. 13-15, 2008.

7. W. Zhou, S. Shao and Z. Gao, "A stability study of the active disturbance rejection control problem by a singular perturbation approach", Applied Mathematical Sciences, vol. 3, No. 10, pp. 491--508, 2009.

8. L. Shao, X. Liao, Y. Xia and J. Han, "Stability analysis and synthesis of third order discrete extended state observer", INFORMATION AND CONTROL-SHENYANG-, vol. 37, No. 2, pp. 135139,2008

9. W. Hui, "Absolute Stability Analysis of Active Disturbance Rejection Controller", Journal of Shanghai University of Electric Power, vol. 27, No. 5, pp. 507-511, 2011.

10. Y. Xia and M. Fu, Overview of ADRC, in Compound Control Methodology for Flight Vehicles. 2013, Springer. p. 21--48.

11. J. Han, "From PID to active disturbance rejection control", Industrial Electronics, IEEE transactions on, vol. 56, No. 3, pp. 900--906, 2009.

12. S. Wang, "ADRC and Feedforward Hybrid Control System of PMSM", Mathematical Problems in Engineering, vol. 2013, No. pp. 12, 2013.

13. [13] Y. YIN, B. ZHENG and H. ZHENG, "A Method for Modeling and Simulation of Brushless DC Motor Control System based on Matlab", JOURNAL OF SYSTEM SIMULATION, vol. 20, No. 2, pp. 293 298, 2008.

14. A. WANG, L. ZHANG and H. LI, "The design and implementation of the high performance permanent magnet synchronous motor servo system", Journal of North China Electric Power University, vol. 38, No. 4, pp. 13-17, 2011. 